\title{
SIMULATION OF ANISOTROPIC WET-CHEMICAL ETCHING USING A PHYSICAL MODEL
}

\author{
J. van Suchtelen ${ }^{1,2}$, K. Sato ${ }^{3}$, E. van Veenendaal ${ }^{2}$, A.J. Nijdam ${ }^{1 *}$, J.G.E. Gardeniers ${ }^{1}$, \\ W.J.P. van Enckevort ${ }^{2}$ and M. Elwenspoek ${ }^{1}$
}

1: MESA Research Institute, Micromechanical Transducers, University of Twente, P.O.Box 217, NL-7500 AE, the Netherlands 2: RIM, University of Nijmegen, the Netherlands, 3: Nagoya University, Japan

emails:jaapvs@sci.kun.nl,sato@everest.mech.nagoya-u.ac.jp,erikvv@sci.kun.nl, a.j.nijdam@el.utwente.nl, j.g.e.gardeniers@el.utwente.nl,wvenck@sci.kun.nl,m.elwenspoek@el.utwente.nl, =ㄹesenting author

\begin{abstract}
We present a method to describe the orientation dependence of the etch rate of silicon, or any other single crystalline material, in anisotropic etching solutions by analytical functions. The parameters in these functions have a simple physical meaning. Crystals have a small number of atomically smooth faces, which etch (and grow) slowly as a consequence of the removal (or addition) of atoms by rows and layers. However, smooth faces have a roughening transition (well known in statistical physics) [1]; at increasing temperature they become rougher, and accordingly the etch and growth rates increase. Consequently, the basic physical parameters of our functions are the roughness of the smooth faces and the velocity of steps on these faces. This small set of parameters describes the etch rate in the two-dimensional space of orientations (on the unit sphere). We have applied our method to the practical case of etch rate functions for silicon crystals in $\mathrm{KOH}$ solutions. The maximum deviation between experimental data and simulation using only nine physically meaningful parameters is less than $5 \%$ of the maximum etch rate. This method, which in this study is used to describe anisotropic etching of silicon, can easily be adjusted to describe the growth or etching process of any crystal.
\end{abstract}

\section{INTRODUCTION}

Wet chemical etching of silicon in anisotropic etchants is used for the fabrication of sensors and actuators. The mechanism of wet chemical etching of silicon is still debated [2-8], while some aspects, as e.g. the emergence of "pseudo" facets in fast etching regions in corners of micromachined structures [9] are not yet completely understood. Computer simulation of wet chemical etching of silicon allows precise compensation of mask patterns and optimization of etchant conditions in order to avoid such undesirable features [10]. To do this, current simulation programs rely on a large database of experimental etch rates for all possible orientations, for a particular set of etchant conditions, without any understanding of the underlying physics and chemistry. In the literature, some methods to extract the full orientation dependence of the etch rate from an experiment are reported, all time-consuming and requiring considerable expertise [11-13]. Ideally, one would have at ones disposal an analytical function of the etch rate as a function of crystal orientation and experimental parameters such as the temperature and the composition of the etchant, based on fundamental knowledge of the etching proces. This allows a more accurate calculation of the shape of an etched structure and also simplifies incorporation of boundary conditions at masks [14][15]. Moreover, if the dependence on the composition of the etchant is cast into an analytical function, calculation of the time evolution of the crystal/etchant interface, including bulk diffusion and convection by gas bubble formation becomes possible.

We have developed a logical construction method which yields such functions [16][17]. Here we will present the results in short.

\section{NETWORK FUNCTIONS}

The central assumption of the network construction principle is that the complete orientation dependence of the etch rate can be reconstructed from the etch rate in the direct vicinity of a limited number of facet orientations. In turn, the etch rate in the vicinity of a facet orientation can be described as an interaction of a number of physical processes on the surface. Intuitively, etching of a crystal can be compared 
with an electrical network. There is a mathematical resemblance between functions used to describe the frequency-reponse behaviour of an electrical network and functions that describe the "orientation-response behaviour" of an etching crystal. The analogy we use is shown in fig.1.

Consider an ideal LC loop (zero resistance). Varying the frequency of an $\mathrm{AC}$ voltage source across the resonance frequency $1 / \sqrt{\mathrm{LC}}$ at constant voltage amplitude, we will measure a current $i(\omega)$ obeying a zero-cusped behaviour (first column in fig.1). This behaviour is analogous with the $R(n)$ function of a faceted crystal, which has a zerominimum at a facet orientation. The roughening transition [2] of a facet corresponds with a transition from the sharp zero-cusp to a smoothed minimum at a positive value. In the electrical case, such a transition can be brought about by a resistive shunt across the LC loop (second column in fig.1).

\begin{tabular}{|c|c|c|}
\hline $\begin{array}{c}\text { THE NETWORK } \\
\text { ANALOGY }\end{array}$ & $\begin{array}{l}\uparrow \mid \begin{array}{c}\text { Electrical case } \\
\text { (j) }\end{array} \\
\omega \rightarrow \text { hor: frequency) } \\
\omega\end{array}$ & $\uparrow_{\mathrm{R}} \frac{\begin{array}{l}\text { crystal etch/growth } \\
\text { (hor: orientation) }\end{array}}{\Phi \rightarrow}$ \\
\hline & $L C$ resonator & $\begin{array}{l}\text { cusp at facet } \\
\text { orientation }\end{array}$ \\
\hline & $\begin{array}{l}\text { resonator } \\
\text { damped by shunt }\end{array}$ & $\begin{array}{l}\text { "roughening" } \\
\text { of facet }\end{array}$ \\
\hline & $\begin{array}{l}\text { series of } \\
\text { resonators }\end{array}$ & more facets \\
\hline & $\begin{array}{l}\text { limitation by } \\
\text { series resistance }\end{array}$ & $\begin{array}{l}\text { limitation by } \\
\text { series mechanism }\end{array}$ \\
\hline
\end{tabular}

Figure 1: Analogy between the electrical frequency response functions and etching orientation-response functions.

Complete roughening implies that the facet orientation vector can no longer be recognized as a special orientation. In the electrical network, shortcircuiting of the LC loop does the same for the resonance frequency. As a faceted crystal has more than one facet, a complete $\mathrm{R}(\mathrm{n})$ function has more than one minimum in the full orientation range. In the electrical network, a series-connection of LC loops, tuned to different frequencies, produces a similar effect (third column in fig.1). An R(n) function can be upward-limited by a series process (diffusion, chemical reaction etc.). This corresponds with a series resistance in the electrical case (fourth column in fig.1).

Altogether, we can construct an electrical network displaying a number of smooth or sharp minima in the $i(\omega)$ function by taking basic resonators ( $=\mathrm{LC}$ loops) as building units, and combine them with resistors in a suitable network. The network corresponds with an analytical function $\mathrm{i}(\omega)$. In quite a similar way, we can construct a symbolic network for an R(n) function. However, as an extension from the electrical case we need the possibility to make the network nonlinear. The important point is that the building units and parameters of the network have an obvious physical meaning that can be described and measured.

We have applied our method to the practical case of etch rate functions for silicon crystals in $\mathrm{KOH}$ solutions [12][18-20]. In this short paper, we can present only the results of just one experiment in order to give an impression of the merits of our simulation. This work will be presented more extensively in [17].

\section{CONSTRUCTING A 3D NETWORK ETCH RATE FUNCTION}

In figure $2 a$ the experimental etch rate is presented for etching of silicon in a $26 \mathrm{w} / \mathrm{w}$ (weight percent) $\mathrm{KOH}$ water solution of $70^{\circ} \mathrm{C}$. The orientation dependence of the etch rate is determined by means of the profile of a large hemispherical specimen before and after etching [11]. Obviously, the etch rate displays a sharp cusp down to almost zero for the $\{111\}$ orientation. Furthermore, the $\{100\}$ orientation corresponds to a shallow minimum in the etch rate. Thus, $\{111\}$ and $\{100\}$ are the facets of silicon for etching in concentrated $\mathrm{KOH}$. One might argue that also the $\{110\}$ orientation qualifies, but we presume that the $\{111\}$ and $\{100\}$ facets determine the behavior of the etch rate around $\{110\}$.

The etch rate in the direct vicinity of a facet is determined by the competition between two etching mechanisms. The first mechanism is the motion of steps on the surface. The second mechanism is the nucleation of mono-layer etch pits, that after their formation spread out, which is why this mechanism is also called the birth-andspread mechanism [21]. Both mechanisms have been observed by Allongue et al. [3][4].

It can be shown [17] that

$R(\alpha)=v_{\text {step }} \cdot \sqrt{\sin ^{2} \alpha+\frac{\varepsilon_{(3)}^{2}}{1-\varepsilon_{(3)}^{2}}} \quad$ (Eq. 1)

where $\alpha$ is the inclination angle with the exact facet. For very small roughness parameters the etch 
a

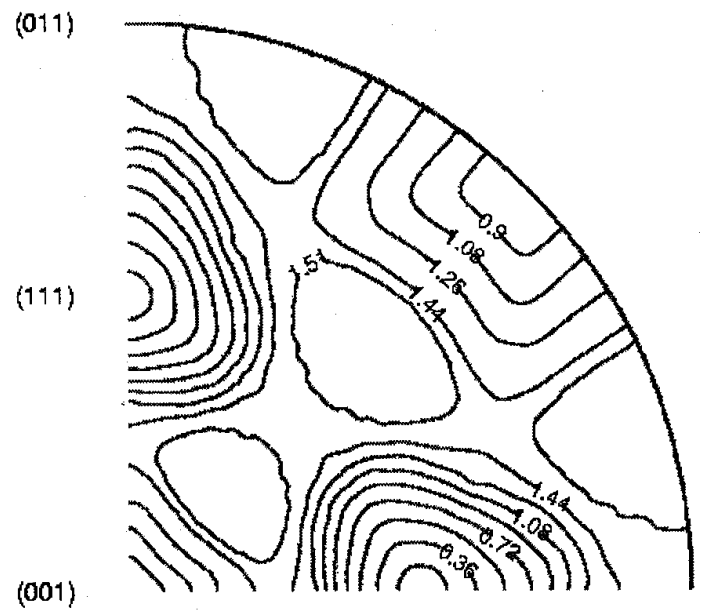

c

(011)

(111)

(001)

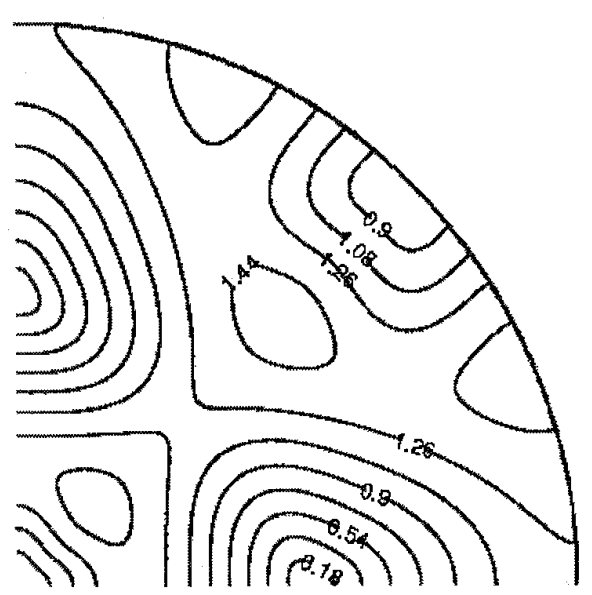

b
(001)

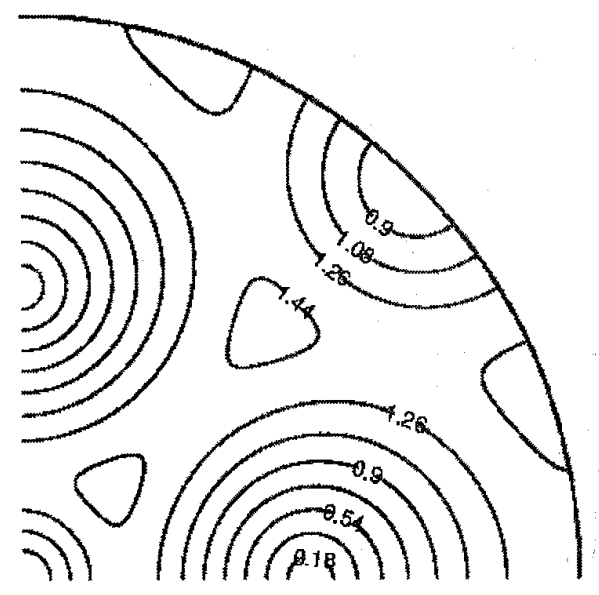

(011)

(111)

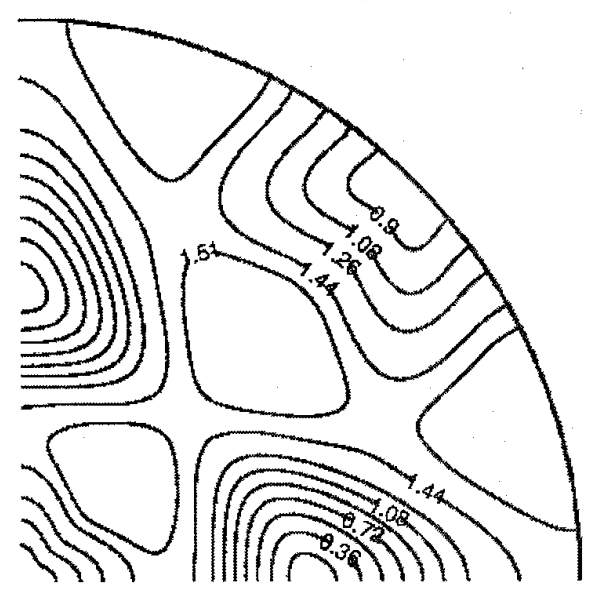

Figure 2: Stereographic representation of

a: the etch result in $26^{\mathrm{w}} /{ }_{w} \mathrm{KOH}$ at $70^{\circ} \mathrm{C}$ (upper left).

$b$ : simulation using 4 parameters (without anisotropy of steps) (upper right).

c: simulation using 6 parameters (with anisotropy of steps) (lower left).

$d$ : simulation using 9 parameters (acounting for corrugational instabilities) (lower right).

rate is given by $v_{\text {step }} \cdot \sin \alpha$ [22]. The step velocity $v_{\text {step }}$ is assumed to be constant and $\varepsilon_{(3)}$, a number between 0 and 1 , is the $3 \mathrm{D}$ roughening parameter, a measure of the frequency of mono-layer etch pit formation. For the $\mathrm{Si}(111)$ facet the birth-andspread mechanism is apparently not important, as the roughening parameter is practically zero, because then we have $R(\alpha)=v_{\text {step }}|\sin \alpha|$ which displays a zero-cusp. For the $\mathrm{Si}(100)$ facet the roughening parameter has a nonzero value, judging by the quite large $(100)$ etch rate $(\alpha=0)$. Thus for $\mathrm{Si}(100)$ the birth-and-spread mechanism apparently plays an important role.

We can now construct a simple network [17], using four phenomenological parameters: $\mathrm{v}_{\mathrm{s} 100}, \mathrm{v}_{\mathrm{s} 111}, \varepsilon_{(3) 100}$ and $\varepsilon_{(3) 111}$, resp. the step velocities and roughening parameters of $\{100\}$ and $\{111\}$. Least squares fitting of these parameters to the experimental etch rate for etching of silicon in 26 ${ }^{\mathrm{w}} / \mathrm{w}$ KOH water solution at $70{ }^{\circ} \mathrm{C}$, yields the 4 parameter fit presented in figure $2 b$. The $4-$ parameter fit shows a qualitative agreement with the experimental data in figure 2a. The minima around $\{100\}$ and $\{111\}$ are visible, as is the saddle point near $\{110\}$. However, the fourfold and threefould symmetry around resp. $\{100\}$ and $\{111\}$ is not reproduced. This is due to the fact that the in reality step velocity is not constant, but depends on the 2D-orientation of the step on the surface. Apperently, step velocity is not isotropic as 
assumed in the simulation.

\section{D-DETAIL IN THE 3D NETWORK ETCH RATE FUNCTION}

It is possible to account for this $2 \mathrm{D}$ anisotropy of the step velocity. The $2 \mathrm{D}$-orientation dependence of the step velocity can be treated in a similar fashion as the 3D-dependence of the etch rate was treated in the previous subsection. First, we identify directions of strong bonds in the surface. These chains of strong bonds are the socalled Periodic Bond Chains (PBC) $[15,23]$. The minima in the step velocity correspond to eps aligned along such a PBC direction. The step velocity for steps almost aligned with a PBC direction is determined by the competition between two processes. The first process is the motion of misorientation kinks. The second process is the nucleation of new kinks. Analogous to equation (1), this competition yields a formula for the step velocity of a step slightly misaligned with a $\mathrm{PBC}$,

$$
v_{s l e p}(\theta)=v_{k i n k} \cdot \sqrt{\sin ^{2} \dot{\theta}+\frac{\varepsilon_{(2)}^{2}}{1-\varepsilon_{(2)}^{2}}}
$$

where $\theta$ is the angle between the 2D-orientation of the step and the vector in the plane perpendicular to the PBC-direction. $v_{\text {kink }}$ is the velocity at which kinks move along a step edge and $\varepsilon_{(2)}$, a number between 0 and 1 , is a measure of the nucleation frequency of kinks.

In the (100) facet two directions of strong bonds can be found, aligned along the [011] and the [01-1] directions. Thus, the step velocity for (100) is expected to be minimal for steps inclined towards a $\{111\}$ facet, which is exactly what can be observed in figure $2 \mathrm{a}$. In the (111) facet three PBC's, forming a perfect triangle, can be found, aligned along the [1-10], [10-1] and [01-1] directions. A complication is that a step aligned along such a PBC inclined towards a $\{110\}$ orientation is a monohydride step, while such a step inclined towards a $\{100\}$ facet is a dihydride step [4]. Inspecting these two kinds of steps, one expects the monohydride steps to be more stable than the dihydride steps. Accordingly, it can be observed in figure $2 \mathrm{a}$ that the step velocity is lower for monohydride steps.

Introducing the $2 \mathrm{D}$ roughening parameters for $\{100\}$ and $\{111\}, \varepsilon_{(2) 100}$ and $\varepsilon_{(2) 111}$, and the kink velocities $v_{k 100}$ and $v_{k 111}$, to our network, we obtain the 6-parameter results presented in figure $2 \mathrm{c}$. It matches the experimental etch rate almost exactly in the vicinity of the $\{111\}$ and $\{100\}$ facets. However, in the region close to $\{110\}$ the agreement is not so good. The remaining deviation between experiment and simulation can be understood from close microscopic inspection of the etched surfaces. Whereas certain regions remain optically smooth, we observe corrugational instabilities around $\{110\}$ [19], while our model predicts a continuously curved surface for all orientations.

\section{AMENDMENTS FOR INSTABILITIES}

Optical microscopy studies of etched silicon surfaces reveal that in the direct vicinity of $\{111\}$, step trains are unstable and bunch forming macro-steps. For larger inclinations towards the $\{110\}$ orientation, this bunching of steps culminates into microfacetting [19]. The physics which causes step trains on the Si $\{111\}$ surface to be unstable is not clear. The observation of step bunching and microfacetting justifies the assumption that the step velocity depends on the inclination angle. Thus, we introduce an ad hoc parameter $C_{111}$ that allows for a $\{111\}$ step velocity that increases with increasing inclination angle. The constant kink velocity $\mathrm{v}_{\mathrm{k} 111}$ is replaced with an inclination dependent kink velocity according to

$v_{k_{111}}=v_{k_{111}}^{0} \cdot\left(1+C_{111}|\sin \alpha|\right) \quad(\mathrm{Eq} .3)$

Optical microscopy has revealed that a surface oriented in a $\{110\}$ direction tends to break up into a staircase structure, also called a zigzag structure [19]. The edges between the zigs and the zags are aligned along [1-10], a PBC direction. The orientation of the zigs and the zags is estimated to be approximately $\{551\}$ [19]. Going from (110) to a (100) facet the zigzag structure persists, but perpendicular to the edges between the zigs and the zags macrosteps become visible. The protruding edges between the zigs and the zags act as so-called velocity sources [14][15]. Velocity sources can be the macroscopically visible results of boundary conditions to the evolution equation of the crystal etchant interface. An edge between a zig and a zag is an example of a boundary. Irrespective of why the protruding edges are formed in the first place, once they are formed some atomic scale etch mechanism dictates the etch rate on these edges. Apparently this dictated etch rate matches the etch 
rate of $\{551\}$, because the zigs and the zags take $\{551\}$ orientations. Perpendicular to the edges between zigs and zags, macrosteps move on the surface. Analogous to the equations for step flow on a crystal facet one expects the contribution of this macrostep motion to equal a constant, a macrostep velocity, times a term giving the density of these macrosteps, i.e. the sine of the inclination to $\{110\}$. As the edges between the zigs and zags are perpendicular to the macrosteps, the velocity source etch rate and the contribution to the etch rate of macrostep motion can simply be added.

Three additional parameters now enter the network function: the ad hoc parameter $\mathrm{C}_{111}$ and the velocity source etch rate $v_{v s}$ plus the macrostep velocity $\mathrm{v}_{\mathrm{ms}}$. In figure $2 \mathrm{~d}$ the 9-parameter fit of the experimental etch rate in figure $2 \mathrm{a}$ is presented. Comparing the fitted and the experimental etch rate we see a good quantitative agreement. The deviation is below $5 \%$ of the maximum etch rate everywhere and for $93 \%$ of the experimental points the deviation is below $3 \%$. Especially if one considers the large roughness in the areas where the fit deviates most, this is quite satisfactory.

\section{DISCUSSION}

We have fitted the 9-parameter network etch rate function to the results of hemisphere etch experiments for $26 \mathrm{w} / \mathrm{w} \mathrm{KOH}$ water solutions for a number of temperatures ranging from 40 to $90{ }^{\circ} \mathrm{C}$. All these experimental etch rates could be fitted with a deviation below $5 \%$ of the maximum etch rate almost everywhere. Merely defining a network etch rate function is only half of the work. The second step of the network construction method for analytical etch rate functions consists of finding the dependence of the phenomenological parameters in the network etch rate function on the experimental parameters. An important concern we have with the existence of a zigzag instability is the fact that, as a consequence of this instability, the $\{110\}$ etch rate may depend on the etching time. In the initial stages of etching there is no staircase structure. Especially then, the etch rate might be different from the etch rate measured in a hemisphere etch experiment.

\section{CONCLUSION}

We have developed a new method to construct analytical functions that accurately describes anisotropic etchrate functions and consist of a limited number of physically meaningful phenomenological parameters.
The advantages of our method are:

- an analytical function with a limited set of parameters is a practical input for a fast simulation algorithm,

- the method enables us to define a continuum function in terms of the experimental parameters as orientation, composition and temperature,

- it is possible to include the etching process mathematically with transport mechanisms as diffusion, viscous flow and chemical reactions which are usually also described by continuum equations

- the method is universal: it works for all single crystalline materials, for etching, dissolution and growth (e.g. in CVD-reactors) and

- the method is based on physical insight and therefore can be used for deeper understanding physical-chemical processes during etching.

\section{ACKNOWLEDGEMENT}

This work was supported by the Dutch Technology Foundation (STW).

\section{REFERENCES}

[1a] P. Bennema, in: 'Handbook of Crystal Growth', Vol I, Ed. D.T.J. Hurle, Elsevier Science

Publishers, Amsterdam (1993) 477

[1b] M. Elwenspoek, J.Electrochem.Soc. 140

(1993) 2075

[2] H. Seidel, L. Csepregi, A. Heutberger and H. Baumgärtel, J. Electrochem. Soc. 137 (1990) 3612 and 3626

[3] P. Allongue, V. Costa-Kieling and H. Gerischer, J. Electrochem. Soc. 140 (1993) 1009 and 1018

[4] J. Kasparian, M. Elwenspoek and P. Allongue, Surface Science 388 (1997) 50

[5] P.M.M.C Bressers, J.J. Kelly, J.G.E. Gardeniers and M.Elwenspoek, J. Electrochem. Soc. 143

(1996) 1744

[6] M. Elwenspoek, J. Electrochem. Soc. 140

(1993) 2075

[7] H. Camon, Z. Moktadir and M. Djafari-

Rouhani, Mat. Science and Eng. B37 (1996) 142

[8] T. Baum and D.J. Schiffrin, J. Electroan. Chem. 436 (1997) 239

[9] H.L. Offereins, K. Kühl and H. Sandmaier, Sensors and Actuators A25-27 (1991) 9

[10] K. Asaumi, Y. Iriye and K. Sato, Proc. IEEE MEMS 1997 (Nagoya, Jan. 1997) 412

[11] A. Koide, K. Sato and S. Tanaka, Proc. IEEE

MEMS 1991 (Nara, Feb. 1991) 216

[12] K. Sato, M. Shikida, Y. Matsushima, T. 
Yamashiro, K. Asaumi, Y. Iriye and M. Yamamoto, Sensors and Actuators A64 (1998) 87

[13] D. Zielke and J. Frühauf, Sensors and Actuators A48 (1995) 151

[14] J. van Suchtelen, A.J. Nijdam and E. van

Veenendaal, 'The velocity source concept', J.Crystal Growth, accepted

[15] A.J. Nijdam, J.W. Berenschot, J.van

Suchtelen, J.G.E. Gardeniers, M.Elwenspoek, Proc. MME'98 (Ulvik in Hardanger, June 1998) 74, submitted to J. Micromech. Microeng.

[16] J. van Suchtelen, 'The Geometry of Crystal Growth' in Morphology of Crystals, Part C, Ed. I. Sunagawa, Terrapub/Kluwer, Tokyo (1995) ISBN 0-7923-3592-9

[17a] J. van Suchtelen and E. van Veenendaal, 'Orientation-dependent crystal growth and etch rate functions: Part I: The nonlinear network as a mathematical tool', submitted to J. Appl. Phys. [17b] J. van Suchtelen and E. van Veenendaal, 'Orientation-dependent crystal growthand etch rate functions. Part II: Physical aspects of the nonlinear network desciption, submitted to J. Appl. Phys. [17c] E. van Veenendaal, J. van Suchtelen, K. Sato, A.J. Nijdam, J.G.E. Gardeniers, W.J.P. van Enckevort and M. Elwenspoek, 'Orientationdependent crystal growthand etch rate functions. Part III: Application to wet chemical etching of silicon in KOH', submitted to J: Appl. Phys.

[18] A. Koide, K. Sato and S. Tanaka, IEEE MEMS 1990, (Napa Valley, Feb. 1990) 216

[19] K. Sato, M. Shikida, T. Yamashiro, M.

Tsunekawa and S. Ito, IEEE MEMS 1998

(Heidelberg, Jan. 1998) 201

[20] K. Sato, M. Shikida, T. Yamashiro, K. Asaumi, Y. Iriye and M. Yamamoto, IEEE MEMS 1998 (Heidelberg, Jan. 1998) 556

[21a] B. Mutaftschiev, in: 'Handbook of Crystal Growth', Vol I, Ed. D.T.J. Hurle, Elsevier Science Publishers, Amsterdam (1993) 187

[21b] A.C. Zettlemyer, Nucleation (Dekker, New York, 1969)

[22] M.C. Elwenspoek, J. Micromech. Microeng. 6 (1996) 405

[23] P. Hartman and W.G. Perdok, Acta Cryst. 8 (1955) 49, 521 and 525 